\title{
Untangling the Potential of Carbon Quantum Dots in Neurodegenerative Disease
}

\author{
Lindsey Jung ${ }^{1}$, Prakash Narayan ${ }^{2, *}$, Sreeprasad T. Sreenivasan ${ }^{3, *} \mathbb{C}$ and Mahesh Narayan ${ }^{3, *} \mathbb{C}$ \\ 1 Tenafly High School, Tenafly, NJ 07670, USA; 21ljung@tenafly.k12.nj.us \\ 2 Department of Preclinical Research, Angion Biomedica Corp., Uniondale, NY 11553, USA \\ 3 Department of Chemistry and Biochemistry, The University of Texas at El Paso, El Paso, TX 79968, USA \\ * Correspondence: pnarayan@angion.com (P.N.); sreenivasan@utep.edu (S.T.S.); mnarayan@utep.edu (M.N.)
}

Received: 18 March 2020; Accepted: 13 May 2020; Published: 18 May 2020

\begin{abstract}
The transitioning of carbon quantum dot (cQD) applications from electrochemistry, catalysis and environmental sensing to biomedicine represents an important milestone in its 15-year history; a bellwether for its yet-unrealized potential in interventional biology, imaging, diagnostics, prophylaxis and therapy. However, despite the significant advances made over the last decade in several areas of the cQD domain, our knowledge of the exact chemical ipseity of cQDs at the Angstrom level remains either in its infancy or is largely ignored. The imminent crossing over of cQDs into biological systems and into the blood-brain barrier demands attention to the critical, yet unmet, need to resolve the inherent heterogeneity in CQD preparations and their separation into purified conformers, to identify the issues associated with potential cytotoxicity as well as to examine their bioavailability. Perhaps most importantly, and ironically neglected as well, is the compelling urgency to obtain an atomicand molecular-level understanding of cQD's interactions with biological receptors; a demand that requires absolute knowledge of its structure, chemistry and aspects therein. The need for the total chemical mapping of cQDs, with Angstrom-level resolution, remains the unrealized cornerstone to tailoring its specificity for its designated use in preclinical and clinical trials.
\end{abstract}

Keywords: carbon quantum dots; heterogeneity; structure

Since the accidental discovery of carbon quantum dots (cQDs) in 2004, a class of nanomaterials with sizes in the range of $2-10 \mathrm{~nm}$, as well as their consequential attractive properties, the field has rapidly expanded to now include cQDs made from neat starting materials such as citrate, urea and diethylamine, or from waste products, biomass and plant-based carbon sources such as watermelon peel, grass, banana and strawberry juice, ginger, garlic, coriander and even cyanobacteria [1].

The expansion of the cQD "library" co-relates with the expansion of its applications relative to inorganic quantum dots (QDs). Due to the higher bio-compatibility (compared to their inorganic counterparts) as well as their excellent photoluminescence, cQDs have found widespread applications in the areas of electrochemical immuno-sensing, biological imaging, theranostics and as fluorescent probes. $\mathrm{cQD}$ s with $\mathrm{sp}^{2}$-hybridized carbons are effective in cancer mitigation [1]. A recent and landmark study has extended the role of cQDs to the ambit of neurodegenerative disorders [2]. Graphene-based quantum dots (GQDs) were able to inhibit the fibrillization of $\alpha$-synuclein, a protein associated with the onset of synucleinopathies such as Parkinson's disease [2]. The study also revealed that GQDs were also able to interact directly with mature fibrils and caused their dissolution-suggesting promise in both prophylaxis and therapeutics.

The transitioning of cQDs from its applications in electronics, catalysis and environmental sensing to the biological realm and across the blood-brain barrier represents an important crossover point. Factors such as toxicity, bioavailability, high homogeneity, side effects, druggability and specificity to 
the disease of interest become important in biomedical applications. In this context, it is important to recognize that the synthesis of CQDs even via the mildest of preparations potentially generates a heterogeneous reaction mixture comprising several distinct cQD and non-cQD constituents [3].

A recent study involving cQD synthesis using $m$-resorcinol as the source resulted in a mixture of at least $21 \mathrm{cQD}$ and non-cQD compounds. The study only reinforced the complexity of cQD chemistry and more importantly, demonstrated the need to unequivocally identify each constituent prior to biomedical use [3]. The resolution of CQD chemical composition is only possible by separating the CQD mixture and then identifying the chemical structure of each CQD constituent (including the assignment of chiral centers). Other factors such as the purity, cytotoxicity, bioavailability and druggability are equally important and need to be determined for each CQD within the ensemble prior to any biological use. A case in point is related to the toxicity issues associated with the chemistry driving CQD product formation. cQDs often produced via the Maillard-like reaction may be toxic and/or mutagenic to cells [4]. The early stage condensation reactions remain the backbone of many cQDs, where the elimination of water between an amino group and a carbonyl group results in the formation of a Schiff base. However, it is likely succeeded by a ketosamine that is the product of an Amadori rearrangement reaction. The degradation of Amadori products to reactive carbonyls such as keto-aldehydes, dicarbonyls and reductones can result in the formation of toxic products such as glyoxal, methylglyoxal, 3-deoxyglucosone (3-DG), hetero-cyclic amines and acryl amides.

Other than toxicity-related issues, an atomic- and molecular-level understanding of cQD-receptor interactions is critical for tailored therapies [5-7]. For example, Graphene quantum dots in alveolar macrophages were analyzed in nuclei, nuclear responses and DNA, and docking studies have been previously reported using the crystal structure of DNA (PDB entry code: 1DJD). $\pi-\pi$ stacking (Figure 1A) interactions play a dominant role in the interactions between AG-QDs and DNA chains as ascertained by molecular docking simulations [5].

In CB1-marijuana (Tetrahydrocannabinol) interactions, $\pi-\pi$ stacking plays a dominant role as well. Therefore, $\mathrm{CQDs}$ targeting receptors within the brain need to engage with specificity and high affinity (Figure 1B,C) as shown in a hypothetical CQD prepared from marijuana and then docked to the receptor [8]. The anticipated results are significant because they permit an in silico-based design and modelling of CQDs onto the receptor. The experimental validation would require the steps in Scheme 1 to be executed.

The need to resolve the specifics of CQD intervention is further illustrated by the aforementioned ability of graphene quantum dots (GQDs) to not only inhibit $\alpha$-synuclein fibrillization but also dissolve mature fibrils of the Parkinson's-disease-associated amyloid protein [2] The noticeably different fibril-inhibiting tendencies of pristine GQDs, nano-graphene oxide and reduced GQDs observed in that study, suggested variations in the mechanism of action and therefore differences in efficacy. Compounding this knowledge gap is the fact that pristine (pure; undoped) GQDs are an ensemble of structures that differ in molecular mass, chemical activity, likely ability to penetrate the blood-brain barrier, toxicity, etc. [1-3,6,7]. In order to further advance GQD and cQD intervention in neurodegenerative disorders, the critical first-step is the resolution, with atomic precision, of the experimental mechanism of their intervention in the disease-specific amyloidogenic trajectory (Scheme 1).

To illustrate this point, consider a typical amyloid-forming landscape involving the transformation of soluble monomeric amyloid into fibrils (Scheme 1). The process involves the generation of toxic intermediates known as oligomers and proto-fibrils that populate the amyloidogenic trajectory. Given their association with the onset and pathogenesis of neurodegenerative disorders, oligomers and protofibrils are established targets in drug-design efforts. Other intermediates that are on-pathway to these toxic species would also qualify as targets for arresting the identifying pathogenesis. There may be dead-end species that are of little interest therapeutically and serve little purpose and only lead to the futile consumption of any cQDs interacting with them. 
The advance of successful small molecules from molecular dynamics simulations to in vitro and in vivo assays and eventually to clinical trials requires (i) establishing the purity of the small-molecule, (ii) the knowledge of its chemical structure, (iii) the dose-response (and therefore knowledge of its molar mass), (iv) determining in which step the molecule may interfere in the trajectory and (v) how the molecule engages the pathogenic target, etc. [Scheme 1]. Based on the mode of action, structure-activity relationships will permit the development of second-generation candidates that may exhibit enhanced inhibition efficacies, better bioavailability, lesser cytotoxicity, etc.

A
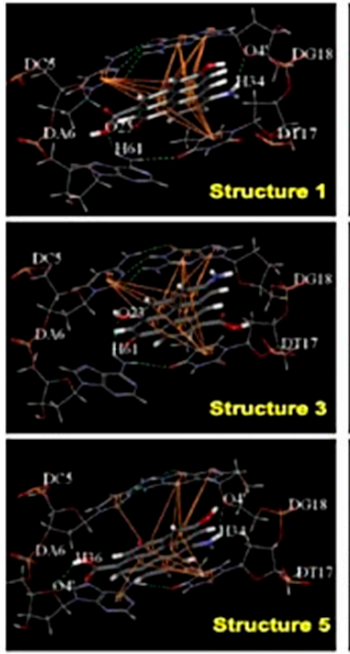

B

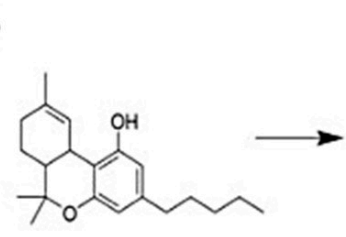

Tetrahydrocannabinol

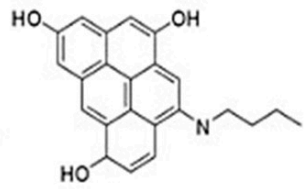

QD 1

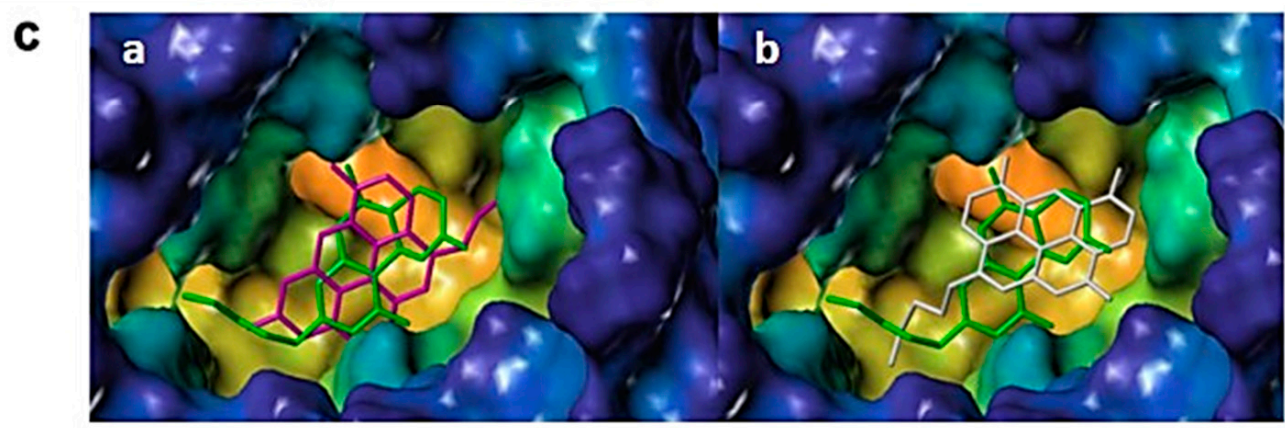

Figure 1. (A) Hydrogen bonding and $\pi-\pi$ interactions between AG-QDs with DNA at select binding sites. Green dotted lines show H-bonds between AG-QDs and basic groups, purines and pyrimidines. Yellow lines show $\pi-\pi$ interactions between AG-QDs and basic groups (cytimidine, guanine, adenine, and thymine). Carbon, oxygen, hydrogen, and nitrogen are colored in grey, red, white, and blue, respectively. (B) Comparison of the structures of Marijuana and designed Quantum dots. The formation of the cQD from marijuana results in a greater number of sp2 carbons. In turn, this will increase the hydrophobicity of the designed ligand and thereby increase lipophilic interactions with the CB1 receptor for hydrophobic binding subsites near CB1. It also not only makes the CQD a better free radical scavenger but also assists in tracking (via fluorescence imaging). (C) Docking of Tetrahydrocannabinol(green), and designed Quantum dots (generated "in silico" to potentially bind to the CB1 receptor) in CB1 receptor a) Tetrahydrocannabinol (green) and QD 1 (pink) b) Tetrahydrocannabinol (green) and QD 1 (white). The "cQD"s were docked through CB1 crystal structure (PDB:5TGZ) using SYBYL-X with Surflex-Dock program. Both QD1 (pink), and QD2 (white) were superimposed very well in CB1 binding site, compared to Tetrahydrocannabinol (green). The pockets were filled such that the free energy reached a minimum value effectively (suggestive of a global minima). A part of this Figure is adapted from [5]. 


\section{Hydrothermal Treatment}

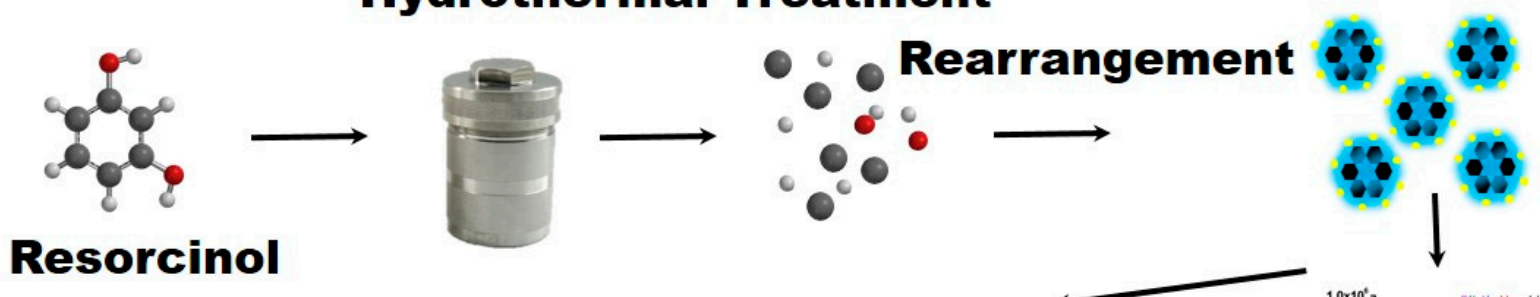

\section{Resorcinol}

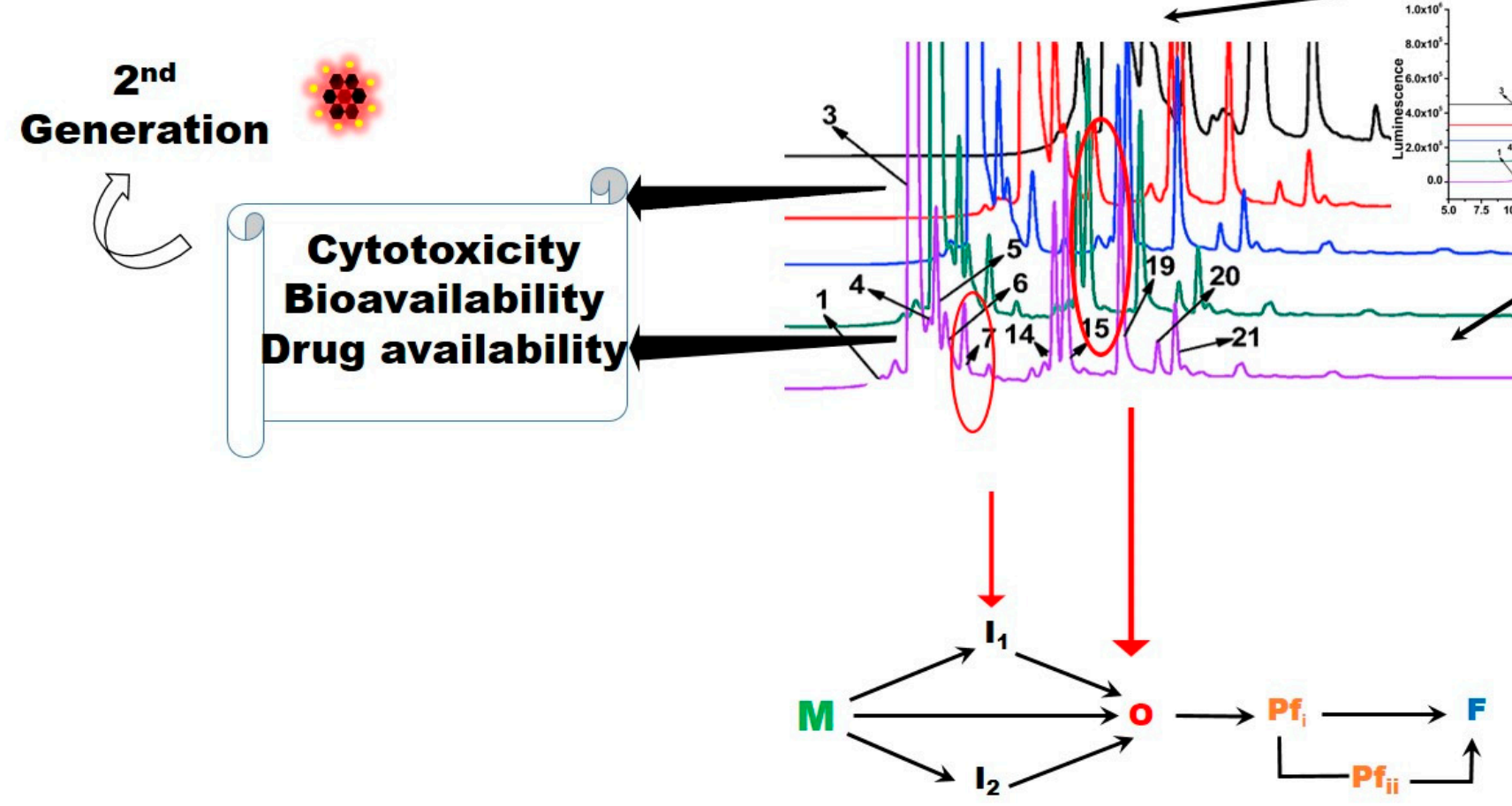

Scheme 1. The preparation, purification (the numbers on the HPLC chromatogram are the order in which the individual compounds in the cQD elute off of the column as "peaks") and application of resorcinol-derived carbon Quantum dots (cQDs). The preparation is followed by the (hypothetical) assessment via the intervention of two distinct cQDs in a typical amyloid-fibril-forming pathway. The next step is the transitioning of the successful cQD to testing in the cell lines via the established bioassays. This cycle is then repeated to facilitate the development of second-generation cQDs. M: Monomer; I: Intermediate; O: Oligomer; Pf: Protofibril; F: Fibril. A part of this Figure is adapted from [3]. 
The holy grail in the transition of cQDs to pre-clinical and clinical trials would be the necessary separation and purification of the $\mathrm{CQD}$ ensembles into individual chemical entities. This would need to be followed by resolving their structure at an atomic level. The mechanism of intervention of the CQD in the desired pathology would need to be addressed. Finally, a toxicological profile of each cQD to be used biomedically must be established. An Angstrom-level characterization would liberate cQDs from their "quantum" confinement and catapult their use across wider domains.

Author Contributions: L.J. did the docking. P.N. supervised and helped interpret the results. S.T.S. and M.N. contributed to the CQD work. All authors have read and agreed to the published version of the manuscript.

Funding: This facility is supported by Grant \#s 2G12MD007592, 5G12MD007592 and 2U54MD007592-26 from the Research Centers in Minority Institutions program of the National Institutes on Minority Health and Health Disparities, a component of the Research Center in Minority Institutions (RCMI) program.

Acknowledgments: The authors would like to thank Angela Lopez for generating Scheme 1 and Gabriela Henriquez for contributing the amyloid pathway to the scheme. SS would like to acknowledge the support through UTEP, and UT STARs. MN acknowledges support from NIH MBRS SCORE 1SC3 GM111200 01A1, the UTEP College of Science (Research Enhancement Award), Holly and Eddie Vazquez (The El Paso Pain Center) and the Border Biomedical Research Center at The University of Texas at El Paso.

Conflicts of Interest: The authors declare no conflict of interest

\section{References}

1. Molaei, M.J. Carbon quantum dots and their biomedical and therapeutic applications: A review. RSC Adv. 2019, 9, 6460-6481. [CrossRef]

2. Kim, D.; Yoo, J.M.; Hwang, H.; Lee, J.; Lee, S.H.; Yun, S.P.; Park, M.J.; Lee, M.; Choi, S.; Kwon, S.H.; et al. Graphene quantum dots prevent $\alpha$-synucleinopathy in Parkinson's disease. Nat. Nanotechnol. 2018, 13, 812-818. [CrossRef] [PubMed]

3. Lu, Y.; Wang, J.; Yuan, H.; Xiao, D. Separation of carbon quantum dots on a C18 column by binary gradient elution via HPLC. Anal. Methods 2014, 6, 8124-8128. [CrossRef]

4. Wei, W.; Xu, C.; Wu, L.; Wang, J.; Ren, J.; Qu, X. Non-Enzymatic-Browning-Reaction: A Versatile Route for Production of Nitrogen-Doped Carbon Dots with Tunable Multicolor Luminescent Display. Sci. Rep. 2014, 4, 3564. [CrossRef]

5. Xu, L.; Dai, Y.; Wang, Z.; Zhao, J.; Li, F.; White, J.C.; Xing, B. Graphene quantum dots in alveolar macrophage: Uptake-exocytosis, accumulation in nuclei, nuclear responses and DNA cleavage. Part. Fibre Toxicol. 2018, 15, 15-45. [CrossRef] [PubMed]

6. Zhou, Y.; Peng, Z.; Seven, E.S.; Leblanc, R.M. Crossing the blood-brain barrier with nanoparticles. J. Control Release 2018, 270, 290-303. [CrossRef] [PubMed]

7. Campbell, E.; Hasan, M.T.; Gonzalez-Rodriguez, R.; Akkaraju, G.R.; Naumov, A.V. Doped Graphene Quantum Dots for Intracellular Multicolor Imaging and Cancer Detection. ACS Biomater. Sci. Eng. 2019, 5, 4671-4682. [CrossRef]

8. Wu, R.; Wang, S.; Zhou, Y.; Long, J.; Dong, F.; Zhang, W. Chromium-Based Metal-Organic Framework MIL-101 Decorated with CdS Quantum Dots for the Photocatalytic Synthesis of Imines. ACS Appl. Nano Mater. 2019, 2, 6818-6827. [CrossRef] 